Instituto Internacional de Investigación y Desarrollo Tecnológico Educativo INDTEC, C.A.

DOI: https://doi.org/10.29394/Scientific.issn.2542-2987.2018.3.10.9.175-195

OAI-PMH: http://www.indteca.com/ojs/index.php/Revista Scientific/oai

Artículo Original / Original Article

\title{
Acciones Preventivas del Deterioro de los Suelos como Alternativa para Fomentar la Cultura Conservacionista
}

Autora: Yoveisy Del Carmen González Izarra Universidad Pedagógica Experimental Libertador, UPEL yove1979@hotmail.com

Barinas, Venezuela

\section{Resumen}

La finalidad de la siguiente investigación, parte de desarrollar acciones preventivas del deterioro de los suelos como alternativa para el fomento de la cultura conservacionista en los habitantes del Caserío La Barinesa, Municipio Bolívar del Estado Barinas, en el periodo 2015-2016. La estrategia de investigación se orienta hacia el paradigma cualitativo. En cuanto al tipo del estudio estará enfocada en una Investigación-Acción Participativa; mientras el diseño se enmarcará en el paradigma cualitativo interpretativo, enfocada en cinco (5) fases o recorrido de ella. En relación con los sujetos de estudio se empleará el universo que lo conformarán dos (02) voceros del Consejo Comunal de la Comisión de Ambiente y dos (02) habitantes y un (01) productor de tabaco. De igual manera, con las técnicas de estudio se basarán en registros narrativos de los fenómenos estudiados, Por lo tanto, la investigación se interactuará con los informantes claves mediante la conversación informal y espontánea. Para la recaudación de la información se trabajará por medio de: la categorización, triangulación y la teorización. Para finalizar se puede establecer que los alcances de la aplicación de un plan para la prevención del deterioro de los suelos por la generación de desperdicios en la cocción del tabaco (residuos tóxicos) como alternativa para el fomento de la cultura conservacionista en los habitantes del Caserío La Barinesa, vendría hacer un trabajo educativo que oriente la población y coadyuve a la formación de las nuevas generaciones.

Palabreas clave: educación ambiental; conservación ambiental; conservación del suelo. 


\title{
Preventive Actions of the Deterioration of Soils as an Alternative to Promote Conservatory Culture
}

\begin{abstract}
The purpose of the following investigation, part of developing preventive actions of the deterioration of soils as an alternative for the promotion of the conservationist culture in the inhabitants of Caserío La Barinesa, Bolívar Municipality of Barinas State, in the period 2015-2016. The research strategy is oriented towards the qualitative paradigm. Regarding the type of study, it will be focused on a Participatory Action Research; while the design will be framed in the interpretative qualitative paradigm, focused on five (5) phases or a tour of it. In relation to the study subjects, the universe will be used, comprising two (02) spokespersons of the Communal Council of the Environment Commission and two (02) inhabitants and one (01) tobacco producer. Similarly, with the study techniques will be based on narrative records of the phenomena studied, Therefore, the research will interact with key informants through informal and spontaneous conversation. For the collection of information will work through: categorization, triangulation and theorization. Finally, it can be established that the scope of the application of a plan for the prevention of soil deterioration by the generation of waste in the cooking of tobacco (toxic waste) as an alternative for the promotion of the conservationist culture in the inhabitants of Caserío La Barinesa, would come to do an educational work that orients the population and contributes to the formation of the new generations.
\end{abstract}

Keywords: environmental education; environmental conservation; soil conservation.

Date Received: 11-01-2018

Date Acceptance: 29-06-2018 


\section{Introducción}

El trabajo educativo debe organizarse en función de las necesidades que involucran a los individuos y en especial a las comunidades, con el propósito de ofrecerle los mejores aportes para lograr el establecimiento de un cambio de conducta favorable ante las diferentes retos que le pueda presentar la vida; para ello, requiere de la aplicación de mecanismos que involucren alternativas de solución a los conflictos de orden social, educativos, ambientales, entre otros; partiendo de una organización ciudadana.

Desde esta perspectiva, la educación y los fundamentos que ella debe aplicar se orientaran en desarrollar, técnicas, métodos y recursos; todos ellos conjugándose en actividades que accionan la gestión escolar en las instituciones educativas, abriendo espacios de participación, con el propósito de relacionarse con el fin último que tiene, como es promover individuos aptos para vivir socialmente y orientados a los conocimientos. De este modo, surgen las estrategias educativas que de acuerdo con Sandoval (2008): dice que son "instrumento curricular para las actividades de enseñanza-aprendizaje generales. Pueden utilizarse para desarrollar actividades y demás contenidos de una destreza en específico; así como las estrategias o bien los recursos que se quieran" (pág. 78).

En ese sentido, Jubero (2008): sostiene que "solo el ser humano es el responsable de la situación de deterioro que sufre el planeta" (pág. 28), y de no tomarse medidas contundentes de inmediato, los daños podrían ser irreversibles y como consecuencia de ello, se tienen los fenómenos climáticos, para confirmar que se está en un punto de riesgo mayor, atentando con el futuro de las generaciones de relevo.

Ante esta situación, la cultura conservacionista no surge del solo hecho de hablar y hacer unos discursos, promesas, publicidad, entre otras actividades que puedan llegar al conglomerado; sino afianzar los aprendizajes a través de la acción por medio del ejemplo, actividades solidarias, búsqueda 
de los causales para medir las consecuencias y aprovechar los aportes aun cuando parezcan los más insignificantes; dado que la concurrencia es importante.

Por su parte, Requena (2017): expresa "la formación ambiental desempeña un papel primordial en la conformación de una conciencia sobre el saber ecológico" (pág. 291), influyendo en el conocimiento que debe tener el ciudadano. De acuerdo a esto, se considera que en Venezuela, por sus características y ubicación geográfica, disfruta de una variedad de condiciones climáticas; no obstante, se viene registrando cambios que han llevado a un macado deterioro por la falta de un comportamiento ambientalista y ante los cuales las personas se muestran indiferentes.

Al respecto Rodríguez (2009): afirma "al ciudadano venezolano no le preocupa o le es de poco interés los problemas de contaminación y deterioro ambiental" (pág. 5). Apoyando lo expuesto por el autor, se puede decir la conducta indiferente ante el desastre de los ríos en su merma continua y de las montañas que son devastadas por la tala o quema indiscriminada, estas son una parte del todo lo que está ocurriendo a escala mundial. Atendiendo a este fenómeno, en las últimas décadas se ha evidenciado un creciente interés social motivado a los problemas y dificultades ambientales a nivel del mundo, causado en su mayoría por las actividades humanas, propias del estilo de desarrollo económico llevado hasta ahora en las ciudades en especial aquellas industrializadas. Por lo que Castillo (2013), destaca:

La pérdida de la calidad ambiental manifestada en la disminución de la diversidad biológica, contaminación de las aguas y del suelo por la presencia de desechos sólidos y líquidos; deterioro de las bellezas escénicas debido a las invasiones o establecimiento de poblados marginales, la expansión demográfica y la poca sensibilización social, entre otros (pág. 45).

Siguiendo el criterio expuesto, existe consenso en que la educación, es 
la alternativa para generar en la población no sólo el conocimiento acerca de los problemas ambientales; el cual se puede lograr a través de una concepción humanista, permitiendo sensibilizar al ciudadano, con el propósito de orientar una conciencia social adaptada al mejoramiento de la calidad de vida; así como también a dar soluciones e implantarse, estando asociado a cierto grado de concienciación promovido desde la educación, mediante la cual las poblaciones pueden apropiarse de un conjunto de estrategias para participar conscientemente en la búsqueda del bien común.

Es por eso que, la contaminación como fenómeno pasa a hacer parte de la vida cotidiana, sin embargo, en los últimos años se ha visto acelerado, aun cuando se hacen esfuerzos a escala mundial, nacional y local para buscar alternativas que contribuyan a optimizar la calidad de vida del planeta y por consiguiente de la población, para evitar contradicciones mayores en la localidad; por el contrario, optimizar la calidad de vida de la población en estudio.

A partir del siguiente estudio se quiere proponer una serie de alternativas que contribuyen a mejorar el ambiente, surgiendo de una sensibilización para la creación de una cultura conservacionista en la población de estudio. Ante lo descrito se considera que en el Caserío La Barinesa, se presenta una problemática desde hace varios décadas, los cuales están relacionadas con las desperdicios generados producto de la actividades agrícolas generadas por los desperdicios que genera el tabaco (residuos tóxicos), los residuos sólidos por exposiciones de los hogares, liquidas por carencia de redes de cloacas, carencia de sistema de recolección de la basura, la tala, quema, entre otros agentes que entorpecen la actividad de resguardo de los suelos.

Por lo antes expuesto, no es menos cierto que si esto permite la generación de ingresos para la población y forma parte de la producción agrícola, también vale destacar que ello trae consigo una serie de problemas 
de índole ambiental que coadyuvan al deterioro del ambiente como es la contaminación de los suelos. Dentro de las observaciones directas de manera informal con los habitantes se pudo conocer que hace varios años el Ministerio del Poder Popular para el Ambiente facilitó información para paliar medianamente este proceso de contaminación, aparte que genera exceso de moscas que contribuyen a los efectos perjudiciales relacionados con la salud de la población en especial con los niños; no obstante, tal situación persiste en la comunidad.

Por tal razón, la situación de contaminación se extiende, puesto que cuando se produce el proceso de perturbación o generan olores fuertes y desagradables se está atentando contra el ambiente; el caso del tabaco después de la cocción produce desperdicios; pero esto no termina allí, motivado que las cantidades que hojas que pasaron por este proceso son arrojadas a cielo abierto provocando esto la proliferación de moscas y roedores; así como también en tiempo de lluvia estos desperdicios son arrastrados por los suelos hasta llegar a los cauces de las aguas naturales de las adyacencias de las comunidades.

En tal sentido, se quiere a través del siguiente estudio sensibilizar para la concreción de una conciencia ciudadana ambientalista que permita de manera eficaz la optimización del ambiente, orientando la población para que sigan las normas sanitarias, se ubiquen los espacios para tales desperdicios afectado lo mínimo posible, que se transformen en facilitadores de mensajes beneficios; puesto que de esta manera se podrá en lo sucesivo evitar desencadenar mayor crisis ambiental y a la misma salud de la población de dicha comunidad.

Cabe destacar que, la investigación se sustentará en basamentos teóricos relacionados con la cultura conservacionista, contaminación de las aguas, los suelos y detrimento de la salud de la población; así como también ofrecer apoyo a la participación comunitaria, tan importante en estos 
momentos que afectan al planeta. Siguiendo el orden de ideas, se considera necesario fundamentar la investigación en teorías que coadyuven a mejorar el ambiente, así como sensibilizar hacia el trabajo de almacenamiento de los residuos tóxicos donde se genere la menor contaminación, daños a la salud y al ambiente, basados de igual manera en la normativa legal que rige el país y convenios internacionales suscritos, como una alternativa que permita optimizar la calidad de vida.

En virtud de lo antes expuesto, la prevención del deterioro de los suelos originado por la generación de desperdicios, busca la recuperación armónica de la interacción entre el ambiente y la actuación de las familias que hacen vida en la comunidad, siendo una alternativa para fomentarse la cultura conservacionista de los habitantes del Caserío La Barinesa, Municipio Bolívar del Estado Barinas, y para ello se hace pertinente plantearse la siguiente interrogante: ¿Es necesario desarrollar acciones preventivas del deterioro de los suelos como alternativa para el fomento de la cultura conservacionista en los habitantes del Caserío La Barinesa, Municipio Bolívar del Estado Barinas?. Por lo antes mencionado se producen las siguientes interrogantes de investigación:

¿Cómo incide en los habitantes en la prevención del deterioro de los suelos en el Caserío La Barinesa, Municipio Bolívar del Estado Barinas?, ¿Qué características presentan las vivencias de los habitantes en el fomento de la cultura conservacionista en su comunidad?, ¿Cómo serán las acciones que permitan la prevención del deterioro de los suelos como alternativa que fomente la cultura conservacionista en los habitantes del Caserío La Barinesa?, ¿Qué estrategias se ejecutarían las acciones que contribuyan con la prevención del deterioro de los suelos como alternativa que permita el fomento de la cultura conservacionista en los habitantes? y ¿Cuáles serían los alcances de la aplicación de un plan para la prevención del deterioro de los suelos como alternativa para el fomento de la cultura conservacionista en los 
habitantes del Caserío La Barinesa?.

Dentro de los propósitos de la investigación se tiene el general, el cual es Desarrollar acciones preventivas del deterioro de los suelos como alternativa para el fomento de la cultura conservacionista en los habitantes del Caserío La Barinesa, Municipio Bolívar del Estado Barinas, en el periodo 20152016; así como también los específicos; destacándose entre ellos: Diagnosticar la incidencia de los habitantes en la prevención del deterioro de los suelos en el Caserío La Barinesa, Municipio Bolívar del Estado Barinas; Caracterizar las vivencias de los habitantes en el fomento de la cultura conservacionista en su comunidad; Elaborar un plan que señale las acciones que permitan la prevención del deterioro de los suelos como alternativa que fomente la cultura conservacionista en los habitantes del Caserío La Barinesa, Municipio Bolívar del Estado Barinas.

Destacándose entre los objetivos específicos también se tiene Ejecutar las acciones que contribuyan con la prevención del deterioro de los suelos como alternativa que permita el fomento de la cultura conservacionista en los habitantes del Caserío La Barinesa, Municipio Bolívar del Estado Barinas y finalmente Evaluar los alcances de la aplicación de un plan para la prevención del deterioro de los suelos como alternativa para el fomento de la cultura conservacionista en los habitantes del Caserío La Barinesa, Municipio Bolívar del Estado Barinas.

Esta iniciativa se fundamenta en un trabajo cooperativo, de sensibilización; así como de la organización de los espacios que hasta el momento carecen de vegetación, lo cual ha provocado la pérdida de especies animales, carencia de plantas; de igual manera la acentuación de calor, exceso de polvo provocado por la brisa que se produce en el lugar, entre otros; lo cual llevado a un cambio en la biodiversidad existente; por lo que se requiere de un cambio para optimizar dichas condiciones. 


\section{Marco Referencial}

De acuerdo a Paredes (2013): en la investigación desarrollada cuyo título es "Programa de sensibilización ambiental para la prevención de los suelos dirigido a los estudiantes de la Segunda Etapa de la Escuela Básica Ciudad de Barinas Sector A-24A del Estado Barinas" (pág. 11), donde el estudio tuvo como objetivo general proponer un programa de sensibilización ambiental para la prevención de los suelos dirigido a los estudiantes de la segunda etapa de la Escuela Básica "Ciudad de Barinas" del Sector A-24A del Estado Barinas para la conservación del ambiente escolar. El mismo se ejecutó a través de proyecto factible apoyado en una investigación de campo con un nivel descriptivo y constó de tres fases: diagnóstico, factibilidad y diseño de la propuesta, la población estuvo conformada por todos los estudiantes pertenecientes a la Segunda Etapa del turno de la mañana y de la tarde de la Escuela Básica Ciudad de Barinas; es decir 333 alumnos, de los cuales se tomó para el estudio una muestra de 120 estudiantes.

El desarrollo del trabajo se realizó a través del estadístico Alpha de Cronbach determinándose bajo esta confiabilidad, asumiendo el protocolo que requiere cada uno de ellos. El cuestionario se fundamentó en los criterios de la escala tipo Likert. Para ello, se diseñó un instrumento de 20 ítems. A través del diagnóstico se pudo comprobar que se deben realizar actividades dirigidas a incrementar la sensibilización y concienciación, tomando en consideración el conocimiento de los principios de conservación en la prevención de los suelos y la comprensión de los problemas ambientales de interés en el país.

Para concluir se puede decir que, con la aplicación del programa de sensibilización ambiental para la prevención de los suelos dirigido a los estudiantes de la segunda etapa permitirá concienciar, motivar y orientar el trabajo hacia el conocimiento de los principales problemas ambientales, tanto a nivel general, como en el ámbito educativo, y su aplicación en la vida diaria a través del desarrollo de buenas prácticas bajo estas acciones de 
conservación.

En relación con los estudio se puede considerar que el mismo permitió apoyar a la investigación que se realiza; orientando la búsqueda de soluciones a través de programas educativos y de sensibilización, con la finalidad de lograr un cambio de actitud en función de mejorar los suelos y por consiguiente la vida en el planeta; en virtud que de acuerdo a lo observado en la comunidad de Corralito I, se carece de sensibilización para proteger el ambiente, resguardar los lugares que le puedan servir de seguridad y esparcimiento; así como de mantener un ambiente agradable, saludable al lograr la inserción de las áreas verdes como alternativa para la protección de la salud, recreación, esparcimiento en el ambiente y cuidado de los suelos.

\subsection{Acciones Preventivas}

Las acciones preventivas contribuyen a prever y solucionar los problemas ecológicos, de relevancia a nivel global, es por ello que cuando el sistema de influencias educativas para la protección del ambiente ha sido correctamente abordado por las escuelas, sociedad, empresas, entre otras; mediante una labor consciente, creadora y entusiasta está es aprovechada de manera significativa; así como también positiva en las prácticas en todos los ámbitos de su vida.

Por ello, se considera que las acciones preventivas son procesos que dura toda la vida y que tiene como principal concienciar el conocimiento ecológico, actitudes, valores, compromisos para acciones y responsabilidades éticas con el propósito de lograr un desarrollo adecuado; así como sustentable. Por ello la dinámica del ambiente y su problemática deben ser asumidas para fortalecer los valores ambientales, de igual manera la participación organizada de la ciudadanía en la solución de problemas de salud pública que afecta a toda la sociedad venezolana; con múltiples problemas del entorno.

A hora bien, ante lo complicado de la problemática ambiental se exige 
al hombre que reaccione y aplique acciones preventivas y pase tal como lo señala Fraire, citado por Boada (2005): evitar una "inmersión sumisa" a la "inmersión crítica" (pág. 12), que conduzca a la conciencia liberadora, por lo que se requiere: el conocimiento de la realidad ambiental y la identificación de sus problemas; la comprensión de los procesos sociales, ecológicos e históricos; el desarrollo de una sensibilidad ambiental y la búsqueda de acciones y medios de acciones disponibles, con el propósito de optimizar la calidad de vida-, así como estabilizar la actividad ambiental en mejora de la población mundial y nacional.

Es necesario hacer referencia a las acciones preventivas con el apoyo de las estrategias, estudios de casos, mapas conceptuales, periódicos murales, visitas guiadas, interpretación de papeles; así como también juegos de simulaciones, estás permiten la formación de un pensamiento crítico, creativo y participativo facilitándole la adquisición de habilidades durante el proceso de su elaboración, para lograr los alcances deseados en el estudio que se lleva acabo y el cual servirá de fundamento a otras investigaciones a ejecutar.

Continuando la idea, se considera que aunado a ello deben impulsar la creatividad y participación, logrando la comprensión de una manera más clara; así como también precisa concisa de los contenidos que se deseen abordar, de igual manera se mantienen atentos e interesados sin perder el hilo de la explicación, facilitando con ello de manera significativa su aprendizaje e igualmente de acuerdo a la dinámica en que se aborda el mismo se mantiene un ambiente en armonía, puesto que logran captar la atención de los involucrado aplicando acciones preventivas en relación con el ambiente.

\subsection{Cultura Conservacionista}

La cultura conservacionista cada vez más está luchando de manera personal, colectiva con las instituciones o empresas que le apuestan a 
fomentar conciencia ambiental con sus prácticas corporativas, esta tendencia se ha venido expandiendo a nivel mundial, aportando acciones más amables con el medio ambiente, buscando crear una cultura conservacionista por parte de las organizaciones, con la finalidad de lograr un mundo mejor.

La Constitución de la República Bolivariana de Venezuela (1999): establece, en su artículo 107, como "obligatoria en los niveles y las modalidades del sistema educativo, así como también en la educación ciudadana no formal"; sin embargo, aún los frutos cognitivos como los concienciadores de la educación ambiental no se han expandido ni recogido debido a las resistencias encontradas en un país cuyas explotaciones mineras y petroleras no sólo le han servido para sustentar su aparato productivo, sino para fortalecer una cultura consumista alejada de accionar las políticas públicas garantes de una efectiva gestión ambiental en pro de la conservación, la defensa, como también mantenimiento del ambiente, en cualesquiera de los estratos gubernamentales.

En la nación, después de la Cumbre de Río de Janeiro en 1992, se crearon un conjunto de leyes en pro del ambiente, siendo necesario conocer una serie de instancias para manejarlo, mientras que la solución de la problemática ambiental fue considerada como una política de estado, por ende, se le otorgó mayor jerarquía a las autoridades ambientales dentro de la administración pública, pero se descuidó la formación y sensibilización ambiental de quienes gerencian en los organismos nacionales, estadales y locales que administran el ambiente, así como a muchos de los educadores encargados de impartir esa disciplina.

En consecuencia, muchos de esos trabajadores no se encuentran identificados con las funciones que ejercen, sólo laboran por la necesidad de obtener un sueldo mensual para sobrevivir. Actúan, por tanto, más como técnicos en la materia que como personas sensibles frente a la problemática ambiental e identificadas con la conservación, la preservación de la naturaleza 
y sus recursos.

\section{Metodología}

La estrategia de investigación se orienta hacia el paradigma cualitativo basado en una investigación acción participativa (IAP); dado que el mismo contempla la observación sistemática y entrevistas con los grupos de estudios que guardan relación con el tema. En este caso, Elliot (2010): define la investigación acción como "el estudio de una situación social para mejorarla" (pág. 84).

En relación con el paradigma cualitativo, es decir, es un método que va a permitir mejorar la realidad de los habitantes del Caserío La Barinesa, Municipio Bolívar del Estado Barinas, con respecto a la implementación de acciones preventivas para evitar el deterioro de los suelos como alternativa para fomentar la cultura conservacionista, incentivando a la autorreflexión de los participantes, para resolver la realidad que están viviendo.

En cuanto al tipo del estudio, la investigación estará enfocada en una Investigación-Acción Participativa, estando asociada al paradigma cualitativo. En este caso, se hace mención a lo expuesto por Salazar (2005): es el "Método de investigación y aprendizaje colectivo de la realidad, basado en un análisis crítico con la participación de los grupos implicados, que se orienta a estimular la práctica transformadora y el cambio social" (pág. 69).

Se seleccionará para conocer la transformación que se realiza y la acentuación en dirección a un fin o un para qué, motivado a su realización sobre la base social, donde pueden intervenir el investigador como parte del estudio, se involucran los aspectos relacionados como la investigación, apoyándola a partir de los testimonios dados.

De acuerdo con las características del estudio, se tomará en consideración el trabajo investigativo realizado por Martínez (2004a), quien explica que cuando se organiza la metodología cualitativa: 
permite descubrir estructuras adecuada con técnicas que pueden consistir en un procedimiento correcto para la realización de las pautas que ella exige, por lo cual se requiere dominio del investigador, para establecer una investigación confiable y fidedigna; que llevará al término que se esperaba entre lo que fue el diseño del modelo y las actividades. (pág. 125).

Por otra parte, con lo expresado, se cumple con las siguientes fases propuestas por Buendía, Colás y Hernández (2008): que se estructura en cinco (05) etapas, entre ellas "Etapa I, Diagnóstico, Etapa II, Planificación, Etapa III, Ejecución, Etapa IV, Evaluación y Etapa V, Sistematización" (pág. 234), las cuales se parafrasean a continuación:

Etapa I, Diagnóstico: Se procede a reconocer de forma clara la problemática presentada, a través de la identificación de las necesidades. El diagnóstico participativo se realiza a través de la entrevista semiestructuradas, como guía para la recolección de información que se aplicó a los involucrados del Caserío La Barinesa, Municipio Bolívar del Estado Barinas.

Etapa II, Planificación: Abarcar el diseño de un plan de trabajo con la finalidad de facilitar la acción, en este caso, se desarrolla en actividades y acciones enmarcadas en técnicas, forma y momento de aplicación adecuadas para la solución a la problemática presentada por la población.

Etapa III, Ejecución: En esta etapa se ejecutan las acciones planificadas dirigidas a desarrollar acciones preventivas del deterioro de los suelos por la generación de desperdicios como alternativa para fomentar la cultura conservacionista en los habitantes del Caserío La Barinesa.

Etapa IV, Evaluación: En la misma se valoran las acciones contentivas en el plan de acción. Cada actividad se evalúa de manera continua con la finalidad de ir reflexionando sobre los procesos emprendidos e ir generando los cambios requeridos o consolidando los logros obtenidos.

Etapa V, Sistematización: Las experiencias se reconstruyeron de todo 
el proceso de trabajo, para luego ser planificadas y presentadas como una alternativa de solución viable.

Para el desarrollo de la investigación, el universo de estudio lo conforman dos (02) voceros del Consejo Comunal de la Comisión de Ambiente y dos (02) habitantes y un (01) productor de tabaco; así como para establecer mayor claridad a este apartado, se indica que los informantes clave la conforman dos (02) voceros del Consejo Comunal de la Comisión de Ambiente y dos (02) habitantes y un (01) productor de tabaco de la comunidad de La Barinesa. Los criterios que se establecidos para la escogencia de los informantes es la siguiente: voceros del Consejo Comunal de la Comisión de Ambiente porque están relacionados con el área de trabajo y dos (02) habitantes y un (01) productor de tabaco como conocedor del área laboral.

En la investigación cualitativa se hacen registros narrativos de los fenómenos estudiados, mediante una serie de técnicas de recolección que darán credibilidad a las futuras interpretaciones utilizando como instrumento se tendrán a: la entrevista en profundidad y la observación participante, con el registro de notas de campo. Para obtener los datos el investigador se incluye en el grupo, hecho o fenómeno observado, para conseguir la información que recoge la información desde afuera, sin intervenir para nada en el grupo social, hecho o fenómeno investigado.

Los procesos de análisis de la información que se utilizarán son: la categorización, triangulación y la teorización. Martínez (2004b): señala que en "la categorización el investigador pone en práctica una actividad mental intensa que lo lleva a clasificar la información en categorías o clases significativas" (pág. 23), según sean las relaciones entre los datos recopilados al efecto. Al respecto, Martínez (2004c), considera que los pasos básicos para la categorización son:

(a) Transcribir en detalle la información primaria o protocolar en 
los dos tercios derechos de las páginas enumeradas, al igual que las líneas; esta numeración facilitará las referencias posteriores; (b) dividir los contenidos en unidades temáticas, es decir, en párrafos o conjunto de párrafos que expresen una idea o concepto central; (c) categorizar con un término o expresión la idea central de cada unidad temática, esta categoría se ubicará en el tercio izquierdo de la página; (d) si hay categorías que se repiten y algunas tienen propiedades o atributos diferentes, se le asigna una subcategoría que puede ser alguna dimensión, atributo, propiedad, condición o consecuencia importante (pág. 126).

El propósito de este proceso será resumir el contenido de la información en conceptos más fáciles de manejar y relacionar, que privilegien lo más valioso de los contenidos primarios, lo que mejor los describa. En el presente estudio, se usará la mencionada técnica para interpretar la información obtenida en función de desarrollar acciones preventivas del deterioro de los suelos. La triangulación, según Cisterna (2003), es:

La acción de reunión y cruce de toda la información pertinente al objeto de estudio, surgida en una investigación por medio de los instrumentos correspondientes y necesarios para recoger la información, que en esencia constituye el corpus de resultados de la investigación (pág. 22).

Por ello, se considera que la información es un acto que se realiza de una vez que ha concluido el trabajo de recopilación de la información. El cruce de la información se efectúa a partir del discurso de los informantes. Haciendo más específico el estudio y la metodología cualitativa, se considera que la atención se orienta en un análisis de la realidad social, por lo que es necesario que la información obtenida en la recolección de datos se maneje de forma sistemática, con el objetivo de generar ideas y establecer, para efectuar una interpretación de los hallazgos. 


\section{Análisis e Interpretación de los Hallazgos}

De acuerdo con las características de la investigación cualitativa se efectúa la interpretación de los hallazgos enunciados por los sujetos implicados en el estudio tal como se nuestra a continuación:

Tabla 1. Subcategoría: Protección ambiental.

\section{Ítem N.․o 1}

¿Cuáles son las estrategias que se le proporcionan para mejorar el nivel de protección de los suelos?

\section{Consejo Comunal - Opiniones}

De lo mencionado por el vocero del Consejo Comunal se evidencio:

- Tomaría en cuenta técnicas nuevas para mejorar el nivel de protección ambiental y en especial de los suelos.

\section{Habitante - Opiniones}

Referente a las estrategias el entrevistado respondió:

1. Serviría de ayuda plantear estrategias para mejorar la protección de los suelos como herramienta para que todas las personas adquieran conciencia de su entorno y puedan realizar cambios en sus valores, conductas y estilos de vidas.

\section{Productor - Opiniones}

El encuestado respondió: Tomaría en cuenta las estrategias nuevas para la protección ambiental de los suelos.

\section{Investigadora - Opiniones}

En lo referido a la protección de los suelos y la utilización de nuevas estrategias que cambiarían su forma de actuar en cuento mejorar el nivel de Protección ambientar: se deduce que tomaría en cuenta las estrategias nuevas para mejorar el nivel de protección ambiental y así ayudar el contexto donde se habita, como herramienta para que todas las personas adquieran conciencia de su entorno, finalmente es importante fomentar el uso de las técnicas, dado que esto serviría para mejorar los suelos, hacer un trabajo sostenible y de autogestión para la comunidad.

Fuente: La Autora (2017).

En atención con la información suministrada en la entrevista se conoció que los voceros del consejo comunal, habitantes y productores mencionaron la necesidad de abocarse al empleo de medios y herramientas para optimizar 
la calidad de los suelos. A lo que Bolívar (2017), comenta:

Se adquiere la formación y sensibilización para la toma de conciencia, así como en el fomento de valores ecológicos, para la puesta en práctica de diversas acciones... consolidación de los mencionados valores, que fomentarán un comportamiento proambiental consustanciado con el mejoramiento de las condiciones de vida en un entorno saludable y sustentable (pág. 3).

Razón por la cual, el estudio parte de la incisiva de fomentar un trabajo organizado que sugiera un cambio de actitud positivo hacia la conservación del ambiente, en especial de los suelos que se encuentran afectados en la comunidad de La Barinesa, motivado al exceso de producción de residuos tóxicos en la cocción del tabaco.

\section{Conclusiones}

En el diagnóstico se espera conocer la incidencia de los habitantes en la prevención del deterioro de los suelos originado por la generación de desperdicios en la cocción del tabaco (residuos tóxicos) en el Caserío La Barinesa, Municipio Bolívar del Estado Barinas; motivado a las consecuencias que ello genera.

Dentro del interés se tiene evitar el deterioro del ambiente manifestado por la constante pérdida de los suelos motivado al pastoreo, desperdicios con residuos sólidos y líquidos, así como de las prácticas agrícolas inadecuadas; la contaminación atmosférica o del medio marino; de igual forma al acelerado uso de los recursos pesqueros, construcciones, la expansión demográfica, entre otros; por lo cual se quiere aplicar un estudio fundamentado en el trabajo directo con la población.

De acuerdo con lo observado, se de evitar el deterioro ambiental como una manera de analizar las causas del deterioro y transformarlas en acciones 
concretas para el desarrollo de medios sostenibles. Para poner fin al uso de combustibles fósiles, se debe recurrir a fuentes de energía alternativas como la energía solar o energía eólica, siendo esta una alternativa de evita la contaminación tan proliferada en la actualidad.

Para finalizar se puede establecer que los alcances de la aplicación de un plan para la prevención del deterioro de los suelos por la generación de desperdicios en la cocción del tabaco (residuos tóxicos) como alternativa para el fomento de la cultura conservacionista en los habitantes del Caserío La Barinesa, vendría hacer un trabajo educativo que oriente la población y coadyuve a la formación de las nuevas generaciones.

\subsection{Recomendaciones}

En cuanto a las consideraciones del estudio se realizan las siguientes recomendaciones:

Efectuar estudios ambientales por el impacto ambiental que pueden generar las construcciones y modificaciones al ambiente.

Incorporar a las comunidades con la finalidad de organizar acciones grupales que vayan en beneficio del ambiente.

Realizar jornadas de sensibilización que orienten hacia a la protección del ambiente a través de programas difusión cultural y educativa ambiental.

\section{Referencias}

Boada, D. (2005). Enseñanza de la Educación Ambiental en el Ámbito Mundial. Mérida, Venezuela: Editorial Piñera.

Buendía, G., Colás, M., \& Hernández, C. (2008). La Investigación Social y Educativa. Colombia: Editorial Secab.

Castillo, S. (2013). Interés social por los Problemas Ambientales. Madrid, España: Alianza Editorial.

Cisterna, L. (2003). Procesos de Cambio Investigativo. Argentina: Editorial 
Trillas.

Constitución de la República Bolivariana de Venezuela (1999). Gaceta Oficial

de la República Bolivariana de Venezuela No. 5.453 Extraordinario de fecha 24 de marzo del 2000. Caracas, Venezuela: Asamblea Nacional Constituyente.

Elliot, M. (2010). La Investigación-Acción en Educación. Caracas, Venezuela: Ediciones Morata S.L.

Jubero, D. (2008). Deterioro del Ambiente. Argentina: Editorial Brujera.

Martínez, A. (2004a,b,c). Ciencia y Arte en la Metodología Investigativa. México: Editorial Trillas.

Paredes, T. (2013). Programa de sensibilización ambiental para la prevención de los suelos dirigido a los estudiantes de la Segunda Etapa de la Escuela Básica Ciudad de Barinas Sector A-24A del Estado Barinas. Barinas, Venezuela: Universidad Nacional Experimental de los Llanos Occidentales Ezequiel Zamora.

Requena, Y. (2017). Investigación Acción Participativa y Educación Ambiental. Revista Scientific, 3(7), 289-308. Recuperado de:

https://doi.org/10.29394/Scientific.issn.2542-2987.2018.3.7.15.289-308

Rodríguez, A. (2009). Problemas de Contaminación y Deterioro Ambiental. Madrid, España: Editorial McGraw-Hill.

Salazar, H. (2005). Método de investigación. Venezuela: Editorial Macmillan de Venezuela, S.A.

Sandoval, G. (2008). Actividades de enseñanza-aprendizaje. Caracas, Venezuela: Editorial Popular.

Bolívar, Y. (2017). Procesos Ambientales y la Sensibilidad Social. Caracas, Venezuela: Editorial Planeta. 


\section{Yoveisy del Carmen González Izarra}

e-mail: yove1979@hotmail.com

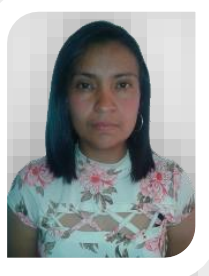

Nacida en Barinitas estado Barinas, Venezuela. Realicé mi educación Superior en el Instituto Universitario de Tecnología "Coronel Agustín Codazzi", Barinas. Obteniendo el título de T.S.U. en Educación Integral; seguidamente realicé la educación Superior en la Universidad Pedagógica Libertador, UPEL, Barinas; donde obtuve le título de Profesora en Educación Integral; en la actualidad me despeño como docente de Aula en el NER 610 de la Parroquia Barinitas estado Barinas, Venezuela. 